\title{
Incidental diagnosis of an adrenal myelolipoma: case report
}

\author{
G. Pamathy ${ }^{1}$, U. Jayarajah ${ }^{1}$, U. S. P. Gooneratne ${ }^{1}$, S. Sivaganesh ${ }^{2}$ \\ 1 University Surgical Unit, National Hospital of Sri Lanka, Colombo, Sri Lanka \\ 2 Department of Surgery, Faculty of Medicine, University of Colombo, Sri Lanka
}

Keywords: Adrenal tumour; myelolipoma; adrenalectomy

\section{Introduction}

Adrenal myelolipomata are rare tumours. A myelolipoma was first reported by Gierke in 1905, and Oberling defined the lesion as myelolipomatoses in 1929 [1]. These lesions were usually detected incidentally, or when symptomatic due to massive growth or hormonal activity. However, the routine use of non-invasive imaging techniques has made incidental diagnosis more common [2].

\section{Case report}

A 58-year-old male was investigated for dyspeptic symptoms. He was a diabetic and hypertensive on treatment. Examination was unremarkable. Abdominal ultrasonography showed a large hyperechoic mass in the right suprarenal gland. Abdominal contrast-enhanced CT scan (CECT) revealed a mass with heterogeneous attenuation measuring $11.48 \times 8.27 \mathrm{~cm}$ (Figure 1) suggestive of a right adrenal myelolipoma.

Serum aldosterone, serum renin and 24 hour urine metanephrine levels were within normal limits indicating the tumour was non-functional. Dehydroepiandrosterone sulphate (DHEAS) levels were not done.

Though asymptomatic, in view of the size of the lesion surgery was recommended. An open transperitoneal right adrenalectomy was performed via a right subcostal incision (Figure 2).

The specimen consisted of a globular soft tissue mass measuring $13 \times 9.5 \times 7 \mathrm{~cm}$ and weighing $500 \mathrm{~g}$. It had yellow and fatty myxomatous areas and microscopic evaluation revealed a mass composed of mature adipose tissue with haematopoietic cells.

The postoperative recovery was uneventful and he was well more than a year after surgery.

Correspondence: S. Sivaganesh

E-mail: drsivgan@yahoo.com

Received: 18-01-2017 Accepted: 14-04-2017

(iD) http://orcid.org/0000-0002-6874-6904

DOI: http://doi.org/10.4038/sljs.v35i1.8354 (cc) BY

The Sri Lanka Journal of Surgery 2017; 35(1): 35-36

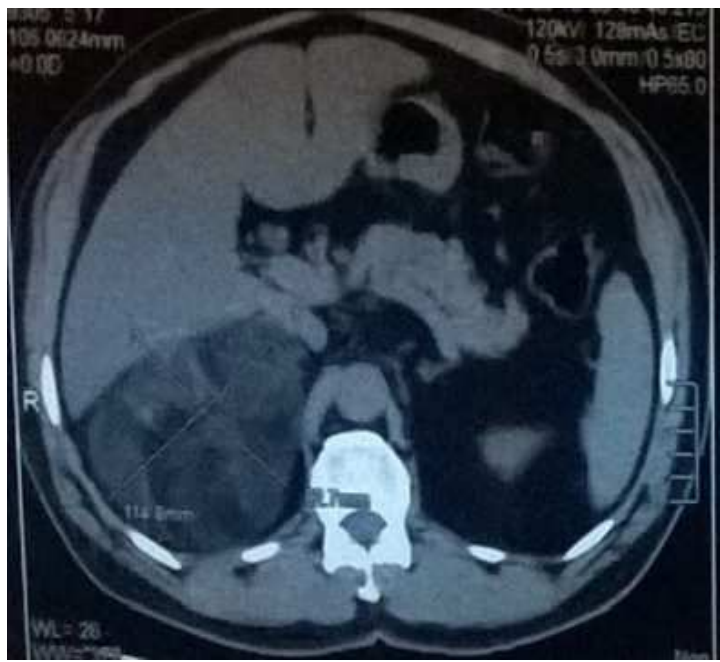

Figure 1. CECT abdomen showing heterogeneous right adrenal lesion

\section{Discussion}

The first operated case of a myelolipoma was reported by Dyckman and Freedman in 1957 . The lesions described were usually less than $4 \mathrm{~cm}$ in diameter while the largest reported tumour weight was $6 \mathrm{~kg}$ [3]. Their incidence ranged from $0.08 \%$ to $0.4 \%$ with involvement of the right adrenal being more common than the left [4].

It is believed that adrenocortical cell metaplasia occurs in these lesions due to stimuli such as inflammation, necrosis, stress or infection [5]. Associations noted with adrenal myelolipomata such as obesity, hypertension and diabetes were present in this patient [6]. They have a relatively slow growth rate with a doubling time of 4.6 to 95.1 months [5].

The commonest symptom is abdominal pain due to tumour haemorrhage, necrosis or compression of adjacent organs [2]. Though usually non-functional, more than 25 cases of endocrine dysfunction associated with myelolipomata have been reported before 2004 [7]. There is a 10\% incidence of associated endocrine conditions such as Conn's syndrome, Cushing's disease and diabetes. There is no potential for malignancy [8].

More than $90 \%$ of adrenal myelolipomata are effectively 
diagnosed by imaging, including ultrasonography, CECT and magnetic resonance imaging (MRI). A CT demonstrating a threshold value of 10 Hounsfield units has a $98 \%$ specificity and $71 \%$ sensitivity for identifying lipid rich adenomas associated with the adrenal gland [9].

Small $(<5 \mathrm{~cm})$, asymptomatic lesions should be observed over a period of 1-2 years [6]. Surgical resection is reserved for symptomatic or larger $(>5 \mathrm{~cm})$ lesions at risk of spontaneous rupture, retroperitoneal haemorrhage and life threatening shock. Laparoscopic adrenalectomy is fast becoming the standard of care though open surgery is appropriate for large lesions as in this case [8]. Early control of the short right adrenal vein is an important step in surgery to prevent significant haemorrhage (Figure 2).

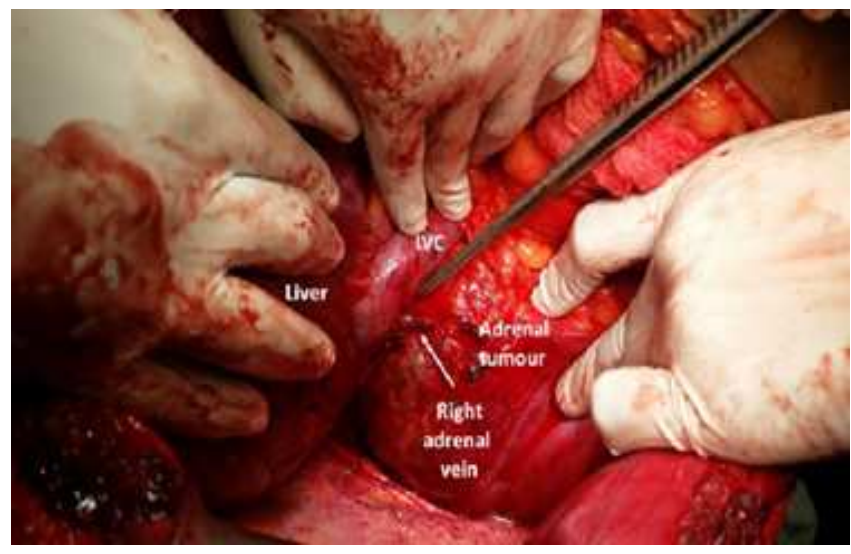

Figure 2. Intra-operative demonstration of right adrenal vein

\section{Conclusion}

Myelolipomas are often incidentally diagnosed adrenal tumours that are usually non-functional and have no malignant potential. Evaluation is by cross sectional imaging and biochemical assessment to exclude secretory function. The majority can be observed without intervention. Surgery is indicated when symptomatic and in large tumours with a potential for spontaneous rupture and major haemorrhage.

\section{References}

1. Lawler L, Pickhardt P. Giant adrenal myelolipoma presenting with spontaneous hemorrhage. CT, MR and pathology correlation. Irish medical journal. 2001;94(8):231-3.

2. Daneshmand S, Quek ML. Adrenal myelolipoma: diagnosis and management. Urology journal. 2009;3(2):71-4.

3. Akamatsu H, Koseki M, Nakaba H, Sunada S, Ito A, Teramoto $\mathrm{S}$, et al. Giant adrenal myelolipoma: report of a case. Surgery today. 2004;34(3):283-5..

https://doi.org/10.1007/s00595-003-2682-4

4. Porcaro A, Novella G, Ficarra V, Cavalleri S, Antoniolli S, Curti P. Incidentally discovered adrenal myelolipoma. Report on 3 operated patients and update of the literature. Archivio italiano di urologia, andrologia: organo ufficiale [di] Società italiana di ecografia urologica e nefrologica/Associazione ricerche in urologia. 2002;74(3):146-51.

5. Meyer A, Behrend M. Presentation and therapy of myelolipoma. International Journal of Urology. 2005;12(3):239-43. https://doi.org/10.1111/j.1442-2042.2005.01034.x

6. Tyritzis SI, Adamakis I, Migdalis V, Vlachodimitropoulos D, Constantinides CA. Giant adrenal myelolipoma, a rare urological issue with increasing incidence: a case report. Cases journal. 2009;2(1):1. https://doi.org/10.4076/1757-1626-2-8863

7. Hisamatsu H, Sakai H, Tsuda S, Shigematsu K, Kanetake H. Combined adrenal adenoma and myelolipoma in a patient with Cushing's syndrome: case report and review of the literature. International Journal of Urology. 2004;11(6):416-8. https://doi.org/10.1111/j.1442-2042.2004.00815.x

8. Puneet TS, Singh S, Kumar M, Shukla V. Adrenal myelolipoma: a case report. The Internet Journal of Third World Medicine. 2006;3.

9. Willatt JM, Francis IR. Radiologic evaluation of incidentally discovered adrenal masses. American family physician. 2010;81(11):1361-6

\section{Key Points:}

- Evaluation by cross sectional imaging and biochemical assessment to exclude secretory function is important in the assessment of adrenal myelolipomata

- Surgery should be considered in large tumours with a risk of spontaneous rupture and haemorrhage 